\title{
Exercícios resistidos no pós- operatório de pacientes submetidos à cirurgia cardíaca
}

\author{
Acadêmico do $5^{\circ}$ ano do curso de Fisioterapia da \\ EBMSP (gahrieltudella@hotmail.com) ${ }^{1}$ (EBMSP - \\ Gabriel Oliveira Tudella \\ Escola Bahiana de Medicina e Saúde Pública - $A v$ \\ Dom João VI 275, - Brotas, 40 290-000 Salvador-BA, \\ Brazil, Telefones: 55-71-3276-8200 ou 55-71-3276- \\ 8261) \\ Fisioterapeuta, MS em Saúde Pública, Docente da \\ Patrícia Alcântara Doval de Carvalho Viana \\ EBMSP da Fundação Bahiana para Desenvolvimento \\ das Ciências \\ Tipo de Pesquisa \\ Origem da Pesquisa \\ Artigo Original \\ Trabalho de Conclusão de Curso de Fisioterapia da \\ Escola Bahiana de Medicina e Saúde Pública. \\ Programa Institucional de Bolsas de Iniciação \\ Apoio financeiro \\ Científica da Fundação Bahiana para \\ Desenvolvimento das Ciências - Ano 2010/2011 - \\ Bolsa \# 1075/2010. \\ Análise do Manuscrito Corpo Editorial da EBMSP \\ Tipo de Análise do Manuscrito \\ Peer Blind Review \\ Recebido em Abr/2011 Aprovado em Jun/2011
}

\section{Resumo}

Introdução: 0 exercício resistido vem proporcionando ao paciente em reabilitação cardíaca um retorno mais precoce às suas atividades funcionais, face aos benefícios oferecidos na utilização dessa modalidade no ambulatório, questiona-se sobre a utilização e segurança desses exercícios na fase hospitalar de reabilitação. 0 objetivo deste estudo foi observar as respostas musculares após exercícios resistidos em pacientes submetidos à cirurgia cardiaca. Métodos: Esse estudo foi um ensaio clínico randomizado composto de 14 pacientes, sendo 8 experimentais e 6 controles. 0 grupo experimental realizou o protocolo de atendimento do Hospital Santa Izabel associado ao treino resistido para membros inferiores e, o grupo controle, manteve o protocolo de rotina do hospital. Resultados: Foi observada diferença estatisticamente significante no grupo estudo em quadríceps direito $(\mathrm{p}=0,05)$, isquiotibial direito $(\mathrm{p}=0,02)$ e esquerdo $(\mathrm{p}=0,04)$ e na distância percorrida $(\mathrm{p}=0,04)$, porém quando comparado a variação intergrupo não houve diferença estatisticamente significante. Conclusão: 0 presente estudo demonstrou que o emprego de exercício resistido na reabilitação cardíaca fase I melhora o grau de força muscular em alguns músculos de forma que esse benefício proporcionará ao paciente um retorno a suas atividades de vida diária de forma mais segura.

Palavras-chave: Reabilitação cardíaca, exercício resistido, fase hospitalar

\footnotetext{
${ }^{1}$ Agradeço aos professores Cloud Kennedy e Roseny Ferreira pela colaboração na elaboração do projeto.
} 


\title{
Resistance exercises in postoperative patients undergoing cardiac surgery
}

\begin{abstract}
Introduction: Resistance exercise has been providing to the patient in a cardiac rehabilitation earlier return to functional activities, given the benefits in using this modality in the clinic, there are questions about the use and safety of these exercises in-hospital rehabilitation. The objective of this study was to observe the responses muscle after resistance exercise in patients undergoing cardiac surgery. Methods: This study was a randomized clinical trial consisting of 14 patients, eight experimental and six controls. The experimental group performed the protocol service of the Hospital Santa Izabel associated with resistance training for lower limbs and the control group maintained the routine protocol of the hospital. Results: We observed a statistically significant difference in the study group in the right quadriceps $(\mathrm{p}=0.05)$, right hamstring $(\mathrm{p}=0.02)$ and left $(\mathrm{p}=0.04)$ and distance $(\mathrm{p}=$ 0.04 ) but when compared to variation intergroup difference was not statistically significant. Conclusion: The present study demonstrated that the use of resistance exercise in phase I cardiac rehabilitation improves the level of muscle strength in some muscles so that this benefit will provide the patient a return to their everyday activities more safely.
\end{abstract}

Keywords: Cardiac rehabilitation, resistance exercise, during hospital stay

\section{Los ejercicios de resistencia en los pacientes postoperatorios de cirugía cardíaca}

\section{Resumen}

Introducción: El ejercicio de resistencia ha estado proporcionando al paciente una rehabilitación cardiaca antes volver a las actividades funcionales, teniendo en cuenta los beneficios en el uso de esta modalidad en la clínica, hay dudas sobre el uso y la seguridad de estos ejercicios en un hospital de rehabilitación. El objetivo de este estudio fue observar las respuestas del músculo después del ejercicio de resistencia en pacientes sometidos a cirugía cardíaca. Métodos: Este estudio fue un ensayo clínico aleatorio que consiste en 14 pacientes, ocho y seis controles experimentales. El grupo experimental realizó el servicio de protocolo del Hospital Santa Izabel asociados con el entrenamiento de resistencia para miembros inferiores y el grupo de control mantuvo el protocolo de rutina del hospital. Resultados: Se observó una diferencia estadísticamente significativa en el grupo de estudio en el cuádriceps derecho $(p=0,05)$, tendón de la corva derecha $(p=0,02)$ e izquierdo $(p=0,04)$ y la distancia $(\mathrm{p}=0,04)$ pero si se compara con la variación diferencia entre los grupos no fue estadísticamente significativa. Conclusión: El presente estudio demostró que el uso de ejercicios de resistencia en la fase I de rehabilitación cardiaca mejora el nivel de fuerza muscular en algunos músculos de modo que este beneficio se proporcionará al paciente el retorno a sus actividades cotidianas con mayor seguridad.

Palabras clave: Rehabilitación cardiaca, el ejercicio de resistencia, durante la estancia hospitalaria

\section{Les exercices de résistance chez les patients subissant une chirurgie cardiaque post-opératoire}

\section{Résumé}

Introduction: des exercices de résistance a fourni au patient une réadaptation cardiaque retour plus rapide aux activités fonctionnelles, compte tenu des avantages dans l'utilisation de cette modalité dans la clinique, il ya des questions sur l'utilisation et la sécurité de ces exercices dans un hôpital de réhabilitation. L'objectif de cette étude était d'observer les 
réponses musculaires après l'exercice de résistance chez les patients subissant une chirurgie cardiaque. Méthodes: Cette étude était un essai clinique randomisé composé de 14 patients, huit contrôles expérimentaux et six. Le groupe expérimental a effectué le service du protocole de l'hôpital Santa Izabel associés à la formation de résistance pour les membres inférieurs et le groupe témoin maintenu le protocole de routine de l'hôpital. Résultats: Nous avons observé une différence statistiquement significative dans le groupe d'étude dans le quadriceps droit $(p=0,05)$, ischio-jambiers droit $(p=0,02)$ et gauche $(p=0,04)$ et à distance $(p=0,04)$ mais lorsqu'on les compare à la variation intergroupe différence n'était pas statistiquement significative. Conclusion: La présente étude a démontré que l'utilisation des exercices de résistance dans la phase I de réadaptation cardiaque améliore le niveau de force musculaire dans certains muscles de sorte que cet avantage sera de fournir au patient un retour à leurs activités quotidiennes de manière plus sûre.

Mots-clés: Réadaptation cardiaque, l'exercice de musculation, cours de l'hospitalisation

\section{Introdução}

As doenças cardiovasculares são um problema de escala global e estima-se que a maior incidência, 80 a $90 \%$ dos casos, ocorra na população de baixa e média renda1. Para combater o avanço dessas enfermidades, estratégias de prevenção e reabilitação vêm sendo tomadas e protocolos são inseridos nos programas de reabilitação cardiovascular.²

Reabilitação cardiovascular é um ramo da cardiologia que envolve uma equipe multidisciplinar a fim de devolver o individuo de forma satisfatória as suas atividades físicas, psicológicas e laborais. Como benefícios pode-se esperar a redução do gasto energético, freqüência cardíaca, pressão arterial e angina. A associação de treinamento físico com mudança no estilo de vida tem demonstrado uma queda na taxa de mortalidade e nos fatores de risco para doenças cardiovasculares33.

Pacientes cardiopatas podem ser direcionados para o tratamento conservador ou cirurgico1. A revascularização do miocárdio e a valyuloplastia são opções para reparo das disfuncões cardíacas. ${ }^{4}$ Após a realização da cirurgia, os pacientes demonstram fraqueza muscular e medo em retornar às suas atividades de vida diária, devido a intensidade da cirurgia cardíaca. 5

Denomina-se exercício resistido a contração muscular contra uma resistência que se opõe ao movimento, que pode ser oferecida com pesos livres, elásticos, aparelhos de musculação, o próprio corpo ou por outros acessórios.?

Exercícios resistidos vêm se tornando cada vez mais utilizados em programas de reabilitação cardiovascular, devido a necessidade das pessoas em retornar às suas atividades funcionais. Faz-se necessário o retorno da resistência e força muscular a níveis satisfatórios para que o paciente realize suas atividades de forma mais econômica do ponto de vista energético. ${ }^{2}$

Antigamente, o treinamento com exercícios resistidos não era considerado seguro para pacientes com doença coronariana ou idosos, hoje sabe-se que o treino isotônico ou isodinâmico com níveis de intensidade baixa e média são fisiologicamente seguros, eficazes e recomendados para o desenvolvimento de força em pacientes cardíacos. ${ }^{6}$

Os exercícios resistidos dinâmicos realizados em paciente hipertensos ou normotensos de intensidade leve a moderada são capazes de reduzir pós-exercício, em média, a pressão arterial sistólica em $3 \%$ e a pressão arterial diastólica em 4\%, sem alterar peso corporal e freqüência cardíaca de repouso.7 
Para mensuração da intensidade do exercício resistido, comumente utiliza-se a avaliação com 1 repetição máxima (1RM) ${ }^{8}$

A utilização de exercícios resistidos tem sido cada vez mais aplicada na recuperação desses pacientes por comprovar a melhora da capacidade cardiopulmonar e força muscular facilitando seu retorno as atividades de vida diária. Além de ser uma terapia de manejo fácil e de baixo custo, os possíveis benefícios advindos dessa pesquisa poderá contribuir para uma redução no tempo de reabilitação desses pacientes.

Face aos benefícios proporcionados pelos exercícios resistidos aos pacientes em reabilitação cardíaca fora do ambiente hospitalar, considera-se relevante avaliar a aplicação desses ainda no período de internamento e observar os efeitos adquiridos.

O objetivo deste estudo foi observar as respostas musculares após exercícios resistidos em pacientes submetidos à cirurgia cardíaca e se existe uma associação da variação do grau de força com o pré-condicionamento e a idade.

\section{Material \& Método}

Esse foi um estudo de ensaio clínico randomizado experimental. A população estudada foi composta por pacientes após cirurgia cardíaca no Hospital Santa Izabel, que se submeteram à cirurgia cardíaca de revascularização do miocárdio e/ou valvuloplastia, de ambos os sexos, com idade maior ou igual a 18 anos. Como critérios de exclusão, os pacientes que estavam instáveis hemodinamicamente (arritmias, hipotensão arterial), que não foram colaborativos, com déficit cognitivo que impediam a compreensão dos comandos na realização dos exercícios e os que sofreram reabordagem cirúrgica.

Os participantes foram randomizados e divididos em dois grupos: Grupo de estudo e grupo controle. O grupo controle participou apenas do tratamento convencional realizado na rotina do Hospital Santa Izabel: exercícios respiratórios de reexpansão e exercícios acróbicos. O grupo estudo participou do tratamento convencional mais o exercício resistido realizado para membros inferiores (MMII).

Antes de iniciar a atividade, foi aferida a tensão arterial (TA), freqüência cardíaca (FC), freqüência respiratória (FR), altura e peso. Foi realizado um teste de caminhada de 6 minutos (TC6') 9 juntamente com a escala de Borg nos dois grupos com objetivo de comparação dos dados.

Foi utilizado o teste de $1 \mathrm{RM}$ para quantificar o peso suportado pelo paciente e a partir desse valor foi estabelecido uma carga de $50 \%$ da aferida, para realizar as 12 repetições, com exceção do exercício para tríceps sural em que foram realizados 2 séries de 12 repetiçôes com peso corporal. Após o término do teste de $1 \mathrm{RM}$ foi aplicado a escala OMNI- RES apenas no exercício de tríceps sural para avaliar a percepção subjetiva de esforço, assim como no final de cada sessão de exercício para este músculo1o.

Depois de avaliada 1RM dos grupamentos musculares eles foram instruídos pelo pesquisador a realizar exercícios para dois grupos musculares por dia de acordo com que já havia sido estabelecido. Os exercícios para quadríceps femoral e isquiotibiais foram realizados em dias alternados com 1 série de 12 repeticoóes e com intervalo de descanso entre as séries de 3 minutos, sendo que primeiro foi realizado o exercício para os grandes grupos musculares seguido dos menores grupos musculares(11,12). Ao término de cada série de exercício para o tríceps sural o paciente foi questionado sobre o nível de dificuldade do exercício na escala OMNI-Res para se comparar a dificuldade do exercício durante o programa.

Os exercícios resistidos foram realizados da seguinte forma; Quadríceps femoral, paciente em sedestação na cama com membros inferiores pendentes ( $90^{\circ}$ de flexão de joelho e quadril), o movimento realizado foi a extensão do joelho unilateral alternando o membro, com peso fixado na região do tornozelo. Isquiotibiais, paciente em ortostase, com 
joelho que realizou o movimento apoiado, foi feito uma flexão do joelho unilateral com peso fixado no tornozelo do paciente, ao término da série o peso é transferido para o membro contralateral13. Tríceps sural foi feito com o paciente em ortostase e realizado plantiflexão a partir da posição neutra da articulação do tornozelo. Se houvesse alguma restrição de amplitude de movimento, dos grupos musculares supracitados ou em caso de dor, o treinamento seria suspenso até a melhora do quadro.

Os dados foram coletados através de uma ficha de avaliação pré-estabelecida.

Como variáveis dependentes do estudo; $1 \mathrm{RM}$, valor da escala OMNI-Res e o teste de caminhada. Como variável independente; exercícios resistidos e co-variavéis; idade, sexo, pré-condicionamento físico e comorbidades associadas.

Para análise da distribuição normal da população foi observado o comportamento da média e da mediana, bem como o teste de kolmogorov-smirnoff. As variáveis não paramétricas do grupo estudo após o exercício resistido como alteração da distância percorrida e força muscular dos músculos quadríceps direito e esquerdo, isquiotibiais direito e esquerdo, tríceps sural, foram analisadas pelo teste de Wilcoxon. Foi realizado o teste Mann-Whitney para análise da variação da força muscular e distância percorrida entre os grupos controle e estudo. Em relação à correlação da idade com as variáveis de variação de força do quadríceps esquerdo e direito, isquiotibial direito e esquerdo, tríceps sural e distância percorrida foi utilizado o teste de correlação de Spearman.

\section{Resultados}

Este estudo avaliou 33 pacientes que se encontravam no pós-operatório imediato de cirurgia cardíaca. Por critérios de exclusão determinados previamente para a realização do estudo, 19 foram excluídos pela falta de informações e/ou desistência do voluntário durante a pesquisa. Para o preenchimento dos grupos, foi feito uma randomização dos pacientes, que ao final do período de estudo atingiu um total de 14 pacientes sendo 8 experimentais e 6 controles. Foi constatada uma distribuição normal da população, de acordo com a Tabela 1.

Foi observada diferença estatisticamente significante no grupo estudo em quadríceps direito $(p=0,05)$, isquiotibial direito $(p=0,02)$ e esquerdo $(p=0,04)$ e na distância percorrida $(p=0,04)$, de acordo com a Tabela 2

Na tabela 3 que apresenta os resultados do grupo controle houve diferenca estatisticamente significantes em quadríceps direito $(\mathrm{p}=0,02)$ e quadríceps esquerdo $(\mathrm{p}=0,04)$.

Em relação à diferença da resistência máxima e distância percorrida entre o grupo controle e estudo, não foi constatado diferença estatisticamente significante para nenhuma das variáveis analisadas assim demonstrado na Tabela 4.

Na Tabela 5 em relação à variação da resistência máxima e distância percorrida no grupo estudo em relação aos praticantes de atividade física, foi observado diferença estatisticamente significante em Quadríceps esquerdo $(\mathrm{p}=0,01)$, Isquiotibial direito $(\mathrm{p}=$ $0,01)$ e esquerdo $(p=0,01)$

Na correlação da idade com a variação de força dos grupo musculares e a distância percorrida, houve diferença significativa nos valores de quadríceps direito $(p=0,02)$ e isquiotibial direito $(p=0,04)$ com forte associação, $r$ maior que 0,7 . De acordo com a Tabela 6.

Não houve diferença estatisticamente significante na análise do grupo estudo em relação à variação da reșistência máxima e distância percorrida entre típo de cirurgia e sexo. 


\section{Discussão}

Este estudo demonstrou que o emprego de exercício resistido na reabilitação cardíaca fase I melhora o grau de força muscular em alguns músculos de forma que esse beneficio proporcionará ao paciente um retorno às suas atividades de vida diária de forma mais segura, já que é comprovado na literatura14 que o paciente necessita retomar a confiança da sua capacidade física após a alta hospitalar.

O resultado dessa pesquisa colabora com outro achado15 em que exercícios de baixa e média intensidade na fase 1 de reabilitação cardíaca são seguros para os pacientes assim como a utilização de escala de percepção de esforço subjetivo é eficaz para controle das variáveis hemodinâmicas.

Diversos artigos6,11 demonstram a eficácia e a segurança da aplicação de exercícios resistidos em pacientes submetidos à cirurgia cardiaca, mas não foi encontrado, até a presente data, nenhum artigo utilizando carga na fase I de reabilitação cardíaca, porem a observação dos pacientes que realizaram o protocolo proposto por este estudo demonstrou a melhora na realização de atividades em relação aos pacientes do grupo controle, que seguiram o protocolo hospitalar convencional, além de nenhum dos integrantes do grupo estudo ter apresentado alguma alteração ou complicação derivada da aplicação de exercício resistido.

Pacientes que realizavam atividade física antes da cirurgia cardíaca apresentaram resultados significantes no ganho de força muscular quando comparados com os que não praticavam atividade física, o que corrobora com outra pesquisa $16 \mathrm{em}$ que pacientes ativos fisicamente obtiveram resultados relevantes em diversos aspectos como tempo de internação hospitalar, complicações no pós-operatório e outras variáveis.

A aplicação do teste de $1 \mathrm{Rm}$ nos pacientes submetidos a reabilitação cardíaca não desencadeou nenhuma alteração cardíaca patológica confirmando a ideia de que é possível utilizar exercícios resistidos na fase I da reabilitação cardiaca17.

São importantes as considerações sobre o medo que influência o paciente no momento de realizar o exercício resistido, seja este devido a cicatriz no local da cirurgia ou por receio em desencadear uma complicação cardiaca, outra pesquisa confirma a importância de familiarizar o paciente com o tipo de exercício para obter melhores resultados.

$O$ resultado da pesquisa poderia ser mais significativo se houvesse um numero amostral maior, mas como observado em alguns estudos15,18, muitos fatores interferem na manutenção do paciente ao longo da pesquisa comprometendo o seu resultado .

\section{Conclusão}

Foi demonstrado em diversos estudos o beneficio que o exercício resistido proporciona ao paciente cardiopata ou hígido11,19 fora do ambiente hospitalar, mas até o presente momento não havia sido realizada nenhuma pesquisa implementando o exercício resistido neste ambiente.

Como limitação desse estudo podemos citar a dificuldade no controle da amostra devido a fatores que fogem a rotina de um atendimento hospitar, como alta inesperada e reações adversas do paciente. Apesar das explanações feitas pelos pesquisadores aos pacientes para realizar a avaliação subjetiva de esforço no exercício para tríceps sural, houve muita dificuldade, pelos pacientes, em quantificar o esforço na escala OMNI-Res.

Com aplicação deste protocolo pioneiro de exercícios resistidos no ambiente hospitalar e a confirmação de ser um método seguro e eficaz para acelerar o retorno do paciente as suas atividades de vida diária, torna-se relevante a aplicação dessa nova modalidade de exercício no ambiente hospitalar. 


\section{Referências}

1- LESSA, A. et al; "Simultaneidade de fatores de risco cardiovascular modificáveis na população adulta de Salvador (BA), Brasil", Revista Panamericana Saúde Pública, 16(2): 131-37. 2004.

2- NEGRÃo, C E \&; BARRETO, A C P. Cardiologia do Exercício: do atleta ao cardiopata. (2nd ed), BarueriSP: Manole (Cap. 14, pp 272-82).

3- UMEDA, I; Manual de Fisioterapia na Reabilitação Cardiovascular. São Paulo-SP: Manole, (Cap 2 pp. 45-46).

4- SARMENTO, G J V. Fisioterapia Respiratória no Paciente Crítico. (2nd Ed), São Paulo-SP: Manole, (Cap. 14, pp. 422-30), 2005.

5- POWERS, S K \& HOWLEY, E T. Fisiologia do Exercício. Teoria e Aplicação ao Condicionamento e ao Desempenho. (5th ed), São Paulo-SP: Manole, (Cap 17, pp. 333 -35), 2005.

6- BERMUDES, A M L M. "Ambulatory blood pressure monitoring in normotensive individuals undergoing two single exercise sessions: resistive exercise training and aerobic exercise training", Arquivo Brasileira Cardiologia, 82(1): pp. 57-64), 2003.

7- POWERS, S K \& HOWLEY, E T. Fisiologia do Exercício. Teoria e Aplicação ao Condicionamento e ao Desempenho. (5th ed), São Paulo-SP: Manole, (Cap 8, pg. 147-51), 2005.

8- MATERKO, W; NEVES, C E B \& SANTOS, E L. “Modelo de predição de uma repetição máxima (1RM) baseado nas características antropométricas de homens e mulheres", Revista Brasileira de Medicina e Esporte, 13(1): Jan-Fev/2007.

9- "American Thoracic Society. ATS statement: guidelines for six-minute walk test", Am J American Journal of Respiratory and Critical Care Medicine, 166: 111-20. 2004.

10- ROBERTSON, R J; et al. "OMNI RPE during resistance exercise", Official Journal of the American College of Sports Medicine, 2003.

11- "Progression Models in Resistance Training for Healthy Adults. Position stand by College of Sports Medicine", Official Journal of the American College of Sports Medicine, 2009.

12- "Exercise in Individuals With and Without Cardiovascular Disease", Official Journal of the American College of Sports Medicine, 2000.

13- DELAVIER, F; Guia dos Movimentos de Musculação: abordagem anatômica. (2nd ed), São Paulo-SP: Manole, (Cap 5, pag 77-96;

14- GONCALVES, FDP et al.; "Avaliação da qualidade de vida pós-cirurgia cardíaca na fase I da reabilitação através do questionário MOS SF-36", Revista Brasileira de Fisioterapia, 10(1): 121-26. 2006.

15- BOTEGA, et al.; "Cardiovascular behavior during rehabilitation after coronary artery bypass grafting", Revista Brasileira Cirurgia Cardiovascular, 25(4): 527-33. 2010

16- NERY, et al.: "Influência da prática da atividade física no resultado da cirurgia de revascularização miocárdica", Revista Brasileira de Cirurgia Cardiovascular, 22(3): 297-302. 2007.

17- DIAS, et al.; "Influência do processo de familiarização para avaliação da força muscular em testes de 1-RM", Revista Brasileira de Medicina do Esporte, 11(1): Jan-Fev/2005.

18- RICARDO, et al.; "Reabilitação cardíaca com ênfase no exercício: uma revisão sistemática", Revista Brasileira de Medicina do Esporte, 12(5): Set-Out/ 2006.

19- WILLIANS, et al; "Resistance exercise in individuals with and without cardiovascular disease: update", Circulation, 116: 572-84. 2007.

20- FARDY, P S. et al. Técnicas do treinamento em Reabilitação Cardíaca. São Paulo-SP: Manole, 2001.

21- MEDIANO, M F F. et al. “Comportamento sub-agudo da pressão arterial após o treinamento de força em hipertensos controlados", Revista Brasileira de Medicina Esporte, 11(6): Nov-Dez/2005. 


\section{Apêndice}

\section{Tabela 1:}

Características clínicas iniciais da população estudada nos grupos Controle e estudo

\begin{tabular}{l|c|c|c}
\hline & $\begin{array}{c}\text { Grupo } \\
\text { controle } \\
(\mathbf{n = 6 )}\end{array}$ & $\begin{array}{c}\text { Grupo } \\
\text { estudo } \\
\mathbf{( n = 8 )}\end{array}$ & $\mathbf{p}$ \\
\hline Idade* $^{*}$ & $58 \pm 8,53$ & $48,7 \pm 11,4$ & 0,73 \\
\hline Sexo masculino** & $3(33 \%)$ & $6(66,7)$ & 0,33 \\
\hline IMC* $^{*}$ & $24,7 \pm 3,77$ & $25,5 \pm 4,53$ & 0,12 \\
\hline Tipo de cirurgia RM** $^{* *}$ & $4(50 \%)$ & $4(50 \%)$ & 0,53 \\
\hline Atividade física prévia** & $1(20 \%)$ & $4(80 \%)$ & 0,19 \\
\hline Tabagista** & $4(57,1 \%)$ & $3(42,9 \%)$ & 0,28 \\
\hline Distância percorrida* & $220 \pm 112$ & $201 \pm 46,6$ & 0,75 \\
\hline
\end{tabular}

oBS: * Média e desvio padrão; ** Porcentagem; RM; Revascularização do miocárdio

Tabela 2

Apresentação da resistência máxima e distância percorrida no grupo estudo $(n=8)$ no momento inicial e final da intervenção

\begin{tabular}{l|c|c|c}
\hline & Inicial* & Final* & p \\
\hline Quadríceps direito** $^{* *}$ & $6(4,25-9,5)$ & $9,5(4,5-14,7)$ & 0,05 \\
\hline Quadríceps esquerdo** $^{*}$ & $8(5,25-9,75)$ & $10,5(5-14,7)$ & 0,16 \\
\hline Isquiotibial direito** & $4(1,35-7,25)$ & $5(2,25-13)$ & 0,02 \\
\hline Isquiotibial esquerdo** & $5(3,25-8)$ & $7,5(4,5-13,2)$ & 0,04 \\
\hline Triceps sural $^{*}$ & $2(0,5-5)$ & $2(0-2,75)$ & 0,27 \\
\hline Distância percorrida1 $^{*}$ & $180(162,2-250)$ & $359(275-410)$ & 0,04 \\
\hline
\end{tabular}

OBS: *Mediana e intervalo interquartil; **Quilogramas; (1)Metros

Tabela 3

Apresentação da resistência máxíma e distância percorrida no grupo controle $(n=6)$ no momento inicial e final da intervenção

\begin{tabular}{c|c|c|c}
\hline & Inicial* $^{*}$ & Final* $^{*}$ & p \\
\hline Quadríceps direito** $^{* *}$ & $6,5(2,75-9)$ & $10(7,5-13)$ & $\mathbf{0 , 0 2}$ \\
\hline Quadríceps esquerdo** $^{*}$ & $6,5(5,5-9)$ & $11,5(7-13,2)$ & $\mathbf{0 , 0 4}$ \\
\hline Isquiotibial direito** $^{*}$ & $3(2,75-8,5)$ & $5,5(4,75-9,25)$ & 0,16 \\
\hline Isquiotibial esquerdo** $^{*}$ & $4,5(3-8,5)$ & $5,5(4-4)$ & 0,16 \\
\hline Triceps sural** $^{*}$ & $5,5(3-6,5)$ & $4(2,25-6,5)$ & 0,35 \\
\hline Distância percorrida1 $^{*}$ & $156(120-352)$ & $330(255-405$ & 0,14 \\
\hline
\end{tabular}

OBS: *Mediana e intervalo interquartil; ${ }^{* *}$ Quilogramas; (1)Metros 
Tabela 4

Diferença da resistência máxima e distância percorrida entre grupos

\begin{tabular}{l|c|c|c}
\hline & Estudo** $^{* *}$ & Controle $^{* *}$ & p \\
\hline$\Delta$ Quadríceps direito1 $^{*}$ & $3,5(0,5-5)$ & $3,5(1,75-6)$ & 0,76 \\
\hline$\Delta$ Quadríceps esquerdo $^{1}$ & $3(-0,25-5)$ & $2(0,75-7)$ & 1 \\
\hline$\Delta$ Isquiotibial direito1 $^{1}$ & $2(1-5)$ & $2,5(0-3,75)$ & 0,84 \\
\hline$\Delta$ Isquiotibial esquerdo1 $^{*}$ & $3,5(0-5,75)$ & $2(0-4)$ & 0,69 \\
\hline$\Delta$ Triceps sural $^{*}$ & $-1(-2,75-0)$ & $0(-2,25-1,25)$ & 0,46 \\
\hline Distância percorrida* $^{*}$ & $150(112,5-$ & $120(-52,5-267)$ & 0,75 \\
\hline
\end{tabular}

OBS: *Metros; **Mediana e intervalo interquartile; (1)Quilogramas

Tabela 5

Variação da resistência máxima e distância percorrida no grupo estudo entre pessoas que praticam atividade física

\begin{tabular}{l|c|c|c}
\hline & Sim & Não & p \\
\hline Quadríceps direito* $^{*}$ & $4,50(2,50-8,75)$ & $1,5(-2,25-4,5)$ & 0,19 \\
\hline Quadríceps esquerdo* $^{*}$ & $5(4,25-8,75)$ & $0,5(-4,75-2)$ & $\mathbf{0 , 0 1}$ \\
\hline Isquiotibial direito* $^{*}$ & $5(3,5-5,75)$ & $1(1-1)$ & $\mathbf{0 , 0 1}$ \\
\hline Isquiotibial esquerdo* $^{*}$ & $5,5(4,25-6)$ & $0(-1,5-2,25)$ & $\mathbf{0 , 0 1}$ \\
\hline Triceps sural* $^{*}$ & $-2,5(-4,5-1,75$ & $0(-1,5-0)$ & 0,29 \\
\hline Distância percorrida** $^{*}$ & $150(100-179)$ & $147(125-170)$ & 1 \\
\hline
\end{tabular}

OBS: * Quilogramas; ** Metros

Tabela 6

Correlação da variação da resistência máxima e da distância percorrida com a idade no grupo estudo

\begin{tabular}{l|l|l}
\hline & \multicolumn{1}{|c|}{$\mathbf{r}$} & $\mathbf{p}$ \\
\hline$\Delta$ Quadríceps direito & $\mathbf{- 0 . 7 7}$ & $\mathbf{0 , 0 2}$ \\
\hline$\Delta$ Quadríceps esquerdo & $-0,31$ & 0,44 \\
\hline$\Delta$ Isquiotibial direito & $\mathbf{- 0 , 7 1}$ & $\mathbf{0 , 0 4}$ \\
\hline$\Delta$ Isquiotibial esquerdo & $-0,60$ & 0,11 \\
\hline$\Delta$ Triceps sural & $-0,17$ & 0,68 \\
\hline$\Delta$ Distância percorrida & $-0,1$ & 0,87 \\
\hline
\end{tabular}

